\title{
Metastatic melanoma cells with BRAF G469A mutation: nab-paclitaxel better than vemurafenib?
}

\author{
Letizia Porcelli ${ }^{1}$ Gabriella Guida ${ }^{2} \cdot$ Stefania Tommasi $^{3} \cdot$ Michele Guida $^{4}$. \\ Amalia Azzariti ${ }^{1}$
}

Received: 24 March 2015 / Accepted: 28 May 2015

(C) Springer-Verlag Berlin Heidelberg 2015

\begin{abstract}
Purpose BRAF G469A is a missense mutation within exon 11 of the BRAF gene resulting in a constitutively activated enzyme frequently associated with MAP kinase cascade signaling activation. No evidence currently exists about its role in determining sensitivity/resistance to BRAF inhibitors, utilized in the treatment of patients carrying BRAF V600 mutations, and to chemotherapy. The newly established metastatic melanoma (MM) cell line MO-1 was characterized for its sensitivity to vemurafenib and nabpaclitaxel, both already utilized for the treatment of MM.

Methods All analyses were carried out by comparing results with those found in MM cells wild type for BRAF or mutated in V600. In addition, cellular effectors were investigated by ELISA kits, western blotting and flow cytometry.

Results The exposure to vemurafenib inhibited MO-1 cell proliferation at concentrations similar to those obtained in vemurafenib-resistant melanoma models, and an explanation of this sensitivity is the strong activation of Erk $1 / 2$
\end{abstract}

Amalia Azzariti

a.azzariti@oncologico.bari.it

1 Clinical and Preclinical Pharmacology Lab, National Cancer Research Centre Istituto Tumori Giovanni Paolo II, Viale O. Flacco, 65, 70124 Bari, Italy

2 Department of Basic Medical Sciences, Neurosciences and Sense Organs, University of Bari, Pizza Giulio Cesare, 70124 Bari, Italy

3 Molecular Genetics Lab, National Cancer Research Centre Istituto Tumori Giovanni Paolo II, Viale O. Flacco, 65, 70124 Bari, Italy

4 Medical Oncology Department, National Cancer Research Centre Istituto Tumori Giovanni Paolo II, Viale O. Flacco, 65, 70124 Bari, Italy and the low expression of MITF. Nab-paclitaxel strongly reduced proliferation of MO-1 cells perhaps for the very low expression level of PMEL17, transcriptionally regulated by MITF and negatively involved in determining sensitivity to taxanes.

Conclusions Thus, the mutation BRAF G469A in MM might be related to a weak effectiveness of therapy with BRAF inhibitors and a promising therapeutic approach may be with nab-paclitaxel.

Keywords Metastatic melanoma - BRAF G469A mutation $\cdot$ Vemurafenib $\cdot$ Nab-paclitaxel $\cdot$ PMEL17

\section{Introduction}

The mutational status of BRAF (B-Raf proto-oncogene serine/threonine-protein kinase) plays a key role in determining the response to BRAF inhibitors in the treatment of metastatic melanoma (MM) with vemurafenib and dabrafenib showing an high efficacy, when the patient has the BRAF V600E mutation but unfortunately with also a quick recurrence of the disease because the establishment of drug resistance phenomena. The melanoma becomes resistant to this class of drugs because of the over-activation of MEK/ERK1/2 pathway or amplification of the MITF or activation of PI3K/Akt/mTOR pathway, etc. [1]. The substitution of glutamic acid for valine at position 600 (BRAF V600E) is up $>90 \%$ of the mutations in this gene within melanoma, with the remainder of mutations largely resulting from other amino acid substitutions at this position, such as V600K, V600D, or V600R and only few in the glycine-rich $\mathrm{P}$ loop of the $\mathrm{N}$ lobe and in the activation segment and flanking regions [2]. BRAF G469A is a missense mutation within exon 11 of the BRAF gene, due 
to a mutation at nucleotide $1406(\mathrm{G} \rightarrow \mathrm{C})$ leading to an amino acid change from glycine to alanine. G469 is part of the tip of a glycine-rich loop that coordinates the active site on BRAF, and its mutation in alanine leads to a constitutively active state of the enzyme with unregulated cell activity. The BRAF G469A mutation, frequently recovers in lung cancer, is rare in melanoma ( $7 \%)$, and uncertain is its association with a more aggressive disease [3, 4].

Literature data evidenced the relationship between the presence of BRAF G469A and the MAP kinase cascade signaling activation [5], and nothing is reported about its role in conferring sensitivity to the BRAF inhibitors, vemurafenib or dabrafenib or the MEK inhibitor trametinib. Moreover, this mutation is responsible for the protein localization also to the mitochondria [6].

In our institute, a patient with MM was treated with fotemustine, but the disease quickly was in progress. After performing a biopsy, from which a cell line was established and named MO-1, the patient was treated with vemurafenib and than with ipilimumab but the prognosis remained poor.

In the newly established cell line MO-1, the mutation G469A in BRAF gene was confirmed and the absence of literature data on the possibility that this mutation can sensitize cells to treatment with BRAF inhibitors, as already happens when the mutation BRAF V600E is present, suggested to check the sensitivity of cells to vemurafenib. Moreover, MO-1 cells were characterized for the sensitivity to nab-paclitaxel (Abraxane), already in phase III clinical trials for the treatment of MM (www.clinicaltrials.gov).

\section{Methods}

\section{Drugs}

Stock solutions of nab-paclitaxel and vemurafenib were prepared at $20 \mathrm{mM}$ in DMSO and stored in aliquots at $-20{ }^{\circ} \mathrm{C}$. Further dilutions were made in medium supplemented with $10 \%$ fetal bovine serum, $2 \mathrm{mM}$ glutamine, 50,000 UL-1 penicillin and $80 \mu \mathrm{M}$ streptomycin.

\section{Cell lines}

The MO-1 cell line was established following the protocol described in [7]. All other MM cell lines were kindly provided by Dr. Guida [8]. LND1 cell line is wild type (wt) for BRAF, Hmel-1 showed BRAF mutation in V600K and MBA72 in V600E both in heterozygosis. All cell lines were wt for NRAS. Cells were cultured in vitro in D-MEM supplemented with $10 \%$ fetal bovine serum, 50,000 UL-1 penicillin and $80 \mu \mathrm{M}$ streptomycin in a humidified incubator at $37^{\circ} \mathrm{C}$ with an atmosphere containing $5 \% \mathrm{CO}_{2}$.

\section{Cell proliferation assay}

Determination of cell growth inhibition was performed treating cells with at least 6 scalar concentrations of the drug, and after 3 days of continuous exposure, the cell growth was measured by the 3-[4,5-dimethylthiazol-2-yl]2,5-diphenyltetrazoliumbromide (MTT) assay. The concentration responsible for $50 \%$ inhibition of cell growth $\left(\mathrm{IC}_{50}\right)$ was calculated as described in [9].

\section{Protein analysis}

\section{Western blot analysis}

Protein extracts were obtained by homogenization in RIPA buffer $(0.5 \mathrm{M} \mathrm{NaCl}, 1 \%$ Triton X100, $0.5 \%$ NP40, $1 \%$ deoxycolic acid, $3.5 \mathrm{mM}$ SDS, $8.3 \mathrm{mM}$ Tris $\mathrm{HCl} \mathrm{pH} \mathrm{7.4,} 1.6 \mathrm{mM}$ Tris base) in the presence of $1 \mathrm{mM}$ phenylmethylsulfonyl fluoride (PMSF). Total proteins were measured and analyzed by western blotting protocol as described in [9]. Expression levels were evaluated by densitometric analysis using Quantity One software (Biorad, Hercules, CA), and $\beta$-actin expression levels were used to normalize the sample values.

\section{Flow cytometry}

MITF and PMEL levels were measured by flow cytometry (FCM) utilizing the same protocol described by Azzariti et al. [10]. All monoclonal antibodies utilized were provided by Cell Signaling-USA and Sigma-Aldrich, St. Louis, MO-USA. A mouse-HRP or fluorescein-conjugated and a rabbit-HRP (Amersham Pharmacia Biotech, Upsala Sweden) were used as secondary antibodies.

\section{Cell cycle analysis}

After two wash steps in ice-cold PBS (pH 7.4), cells were fixed in $4.5 \mathrm{ml}$ of $70 \%$ ethanol and stored at $-20{ }^{\circ} \mathrm{C}$. For the analysis, the pellet was resuspended in PBS containing $1 \mathrm{mg} / \mathrm{ml}$ RNase, $0.01 \% \mathrm{NP} 40$ and $50 \mu \mathrm{g} / \mathrm{ml}$ propidium iodide (PI) (Sigma). After an incubation time of $1 \mathrm{~h}$ in ice, cell cycle determinations were performed using a FACScan flow cytometer (Becton Dickinson), and data were interpreted using the CellQuest software, provided by the manufacturer.

\section{Cell apoptosis assay}

The Annexin V-FITC Apoptosis Detection Kit (BD Biosciences) was used to detect apoptosis by flow cytometry. Cells were exposed to the drug, and after 24-72 h, they were harvested (including detached cells) and processed according to the manufacturer's instructions. 


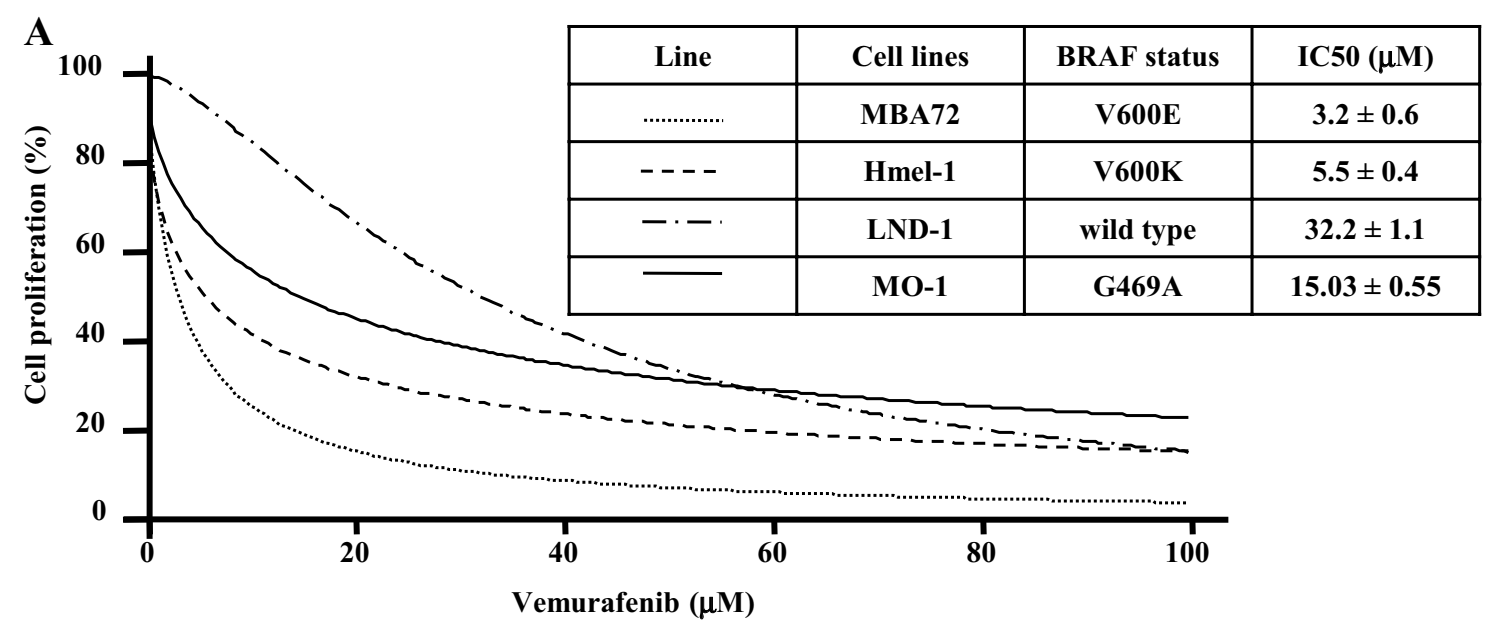

B

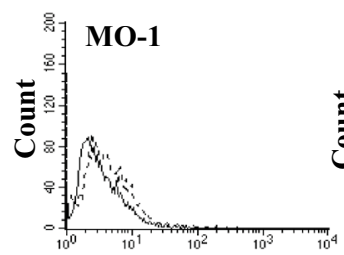

FL-1

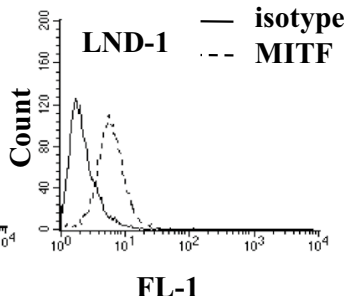

FL-1

C

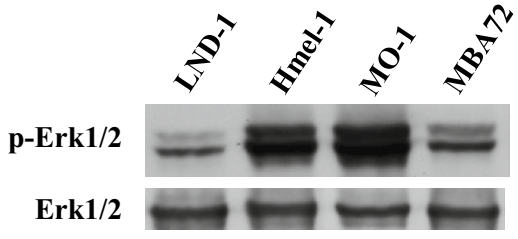

Fig. 1 MO-1 cells and vemurafenib. a MO-1, LND-1, MBA72 and Hmel-1 cells were exposed to vemurafenib $(0.1 \mathrm{nM}-100 \mu \mathrm{M})$ for 3 days, and then, cell growth and $\mathrm{IC}_{50}$ were evaluated as described in M\&M section. b MITF expression in all cells was evaluated by flow cytometry. The histograms of MO-1 and LND-1 cells are showed,

Experiments were performed according to manufacturer's instructions.

\section{Membrane depolarization assay}

Changes in mitochondrial membrane potential were estimated using fluorescent probe JC- 1 . A total of $5 \times 10^{5}$ cells were stained for $20 \mathrm{~min}$ with $1 \mu \mathrm{g} / \mathrm{ml} \mathrm{JC}-1$ (Molecular
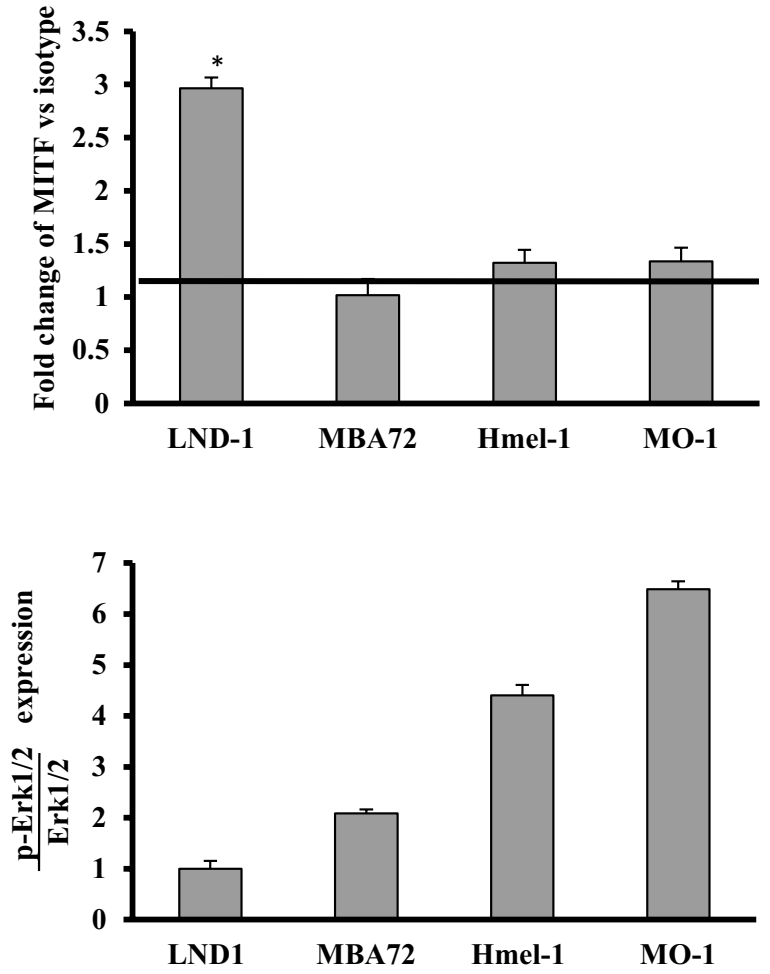

and the quantification of results is reported in the plot in which the MITF levels of the BRAF wt LND-1 cells are statistically different from the others $\left({ }^{*} p<0.01\right)$. $\mathbf{c}$ In all MM cells, the expression levels of p-Erk1/2 and Erk1/2 were determined by western blotting and the results from the densitometric analysis are reported in the plot

Probes) at $37{ }^{\circ} \mathrm{C}$, and then, after 2 wash steps in PBS, cells were analyzed by FCM, collecting JC-1 green fluorescence (FL1) and JC-1 orange fluorescence (FL2).

\section{Statistical analysis}

All in vitro experiments were performed in triplicate, and results have been expressed as the mean \pm standard 
deviation (SD), unless otherwise indicated. $p$ values lower than 0.05 were considered significant. Statistical analyses were performed using the GraphPad Prism software package version 5.0 (GraphPad Software Inc., San Diego, CA, USA).

\section{Results}

The significance of the mutation G469A in BRAF gene in inducing drug response in MM models was determined by comparing the results obtained in MO-1 cells to those obtained in the other MM cells, BRAF wt or mutated. The exposure to vemurafenib for 3 days was responsible for a progressive inhibition of MO- 1 cell growth as a function of drug concentration (Fig. 1a, dashed line). MO-1 (BRAF G469A) cells have the $\mathrm{IC}_{50}$ higher than those measured in BRAF mutated cells and lower than in BRAF wt cells (Fig. 1a). Thus, the BRAF mutation G469A seems to confer a partial resistance to BRAF inhibitors. In support of our hypothesis, the patient, naïve for the treatment with

A

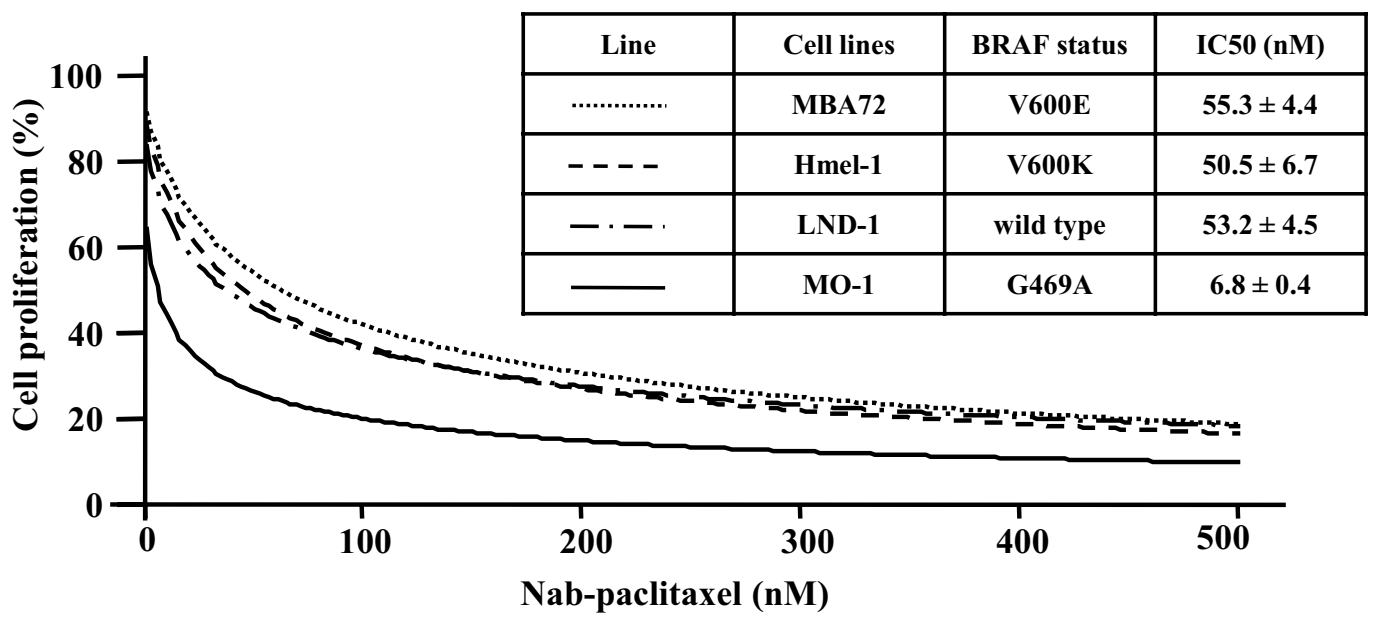

B
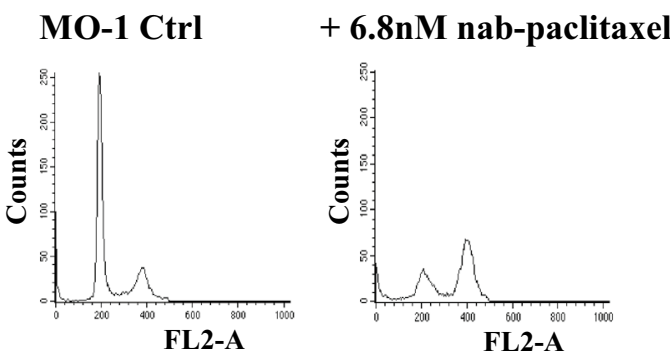

D

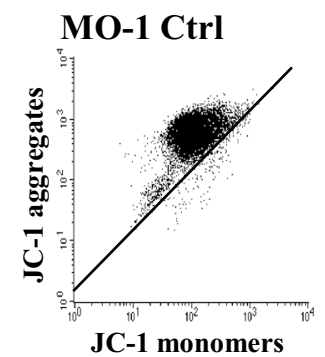

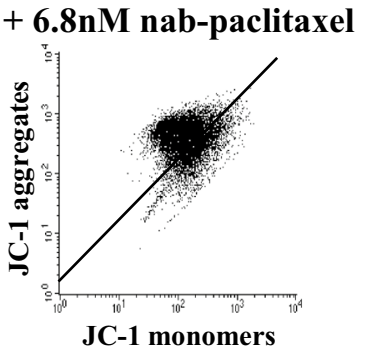

Fig. 2 MO-1 cells and nab-paclitaxel. a MO-1, LND-1, MBA72 and Hmel-1 cells were exposed to nab-paclitaxel $(0.5-500 \mathrm{nM})$ for 3 days, and then, cell growth and $\mathrm{IC}_{50}$ were evaluated. b Histograms of MO-1 cell cycle perturbation by nab-paclitaxel exposure are showed. c Apoptosis induction in MO-1 cells by exposure to

C
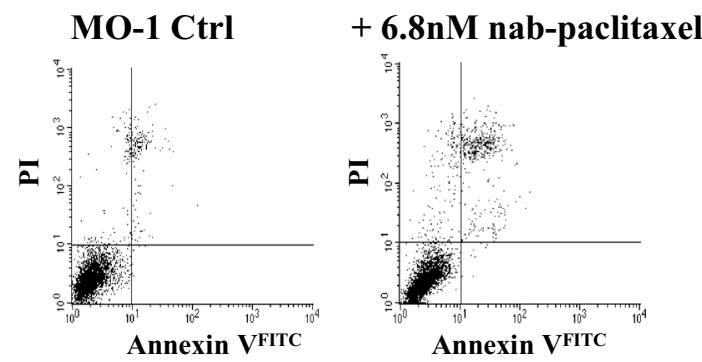

$\mathbf{E}$

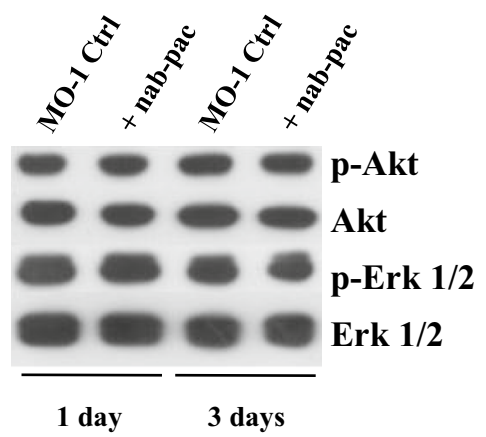

nab-paclitaxel is showed. The analysis was performed by utilizing PI/Annexin V assay. d In MO-1, the depolarization of mitochondria was evaluated by analyzing the change in fluorescence of JC-1 dye. e Immunoblotting of p-Erk, Erk1/2, p-Akt and Akt on MO-1 cells treated with $6.8 \mathrm{nM}$ nab-paclitaxel for 1 and 3 days 
vemurafenib, after the relapse of the disease, was treated with this BRAF inhibitor showing a partial response to the treatment.

To investigate the cellular pathway(s) responsible for the constitutive resistance to vemurafenib related to this mutation, the expression levels of Erk1/2 or MITF, often altered in vemurafenib-resistant MM models, were analyzed [1]. In Fig. 1b, c, the absence of the amplification of MITF is showed together with a marked activation of Erk1/2; in fact, $\mathrm{p}$-Erk1/2 level is higher than those found in all other cell lines, while MITF was similar to the BRAF mutated cells, suggesting the relevant role of the activation of the proliferation pathway in determining a low response to BRAF inhibitors [11].

The transient positive responses of the patient treated before with fotemustine and after with vemurafenib have highlighted the need to find a different and effective therapeutic option.

The effectiveness of the nab-paclitaxel was investigated; this drug is a new albumin-bound form of paclitaxel, already approved for breast cancer and in clinical trials for the treatment of MM patients [12]. MO-1 cells were treated with nab-paclitaxel for 3 days with surprising results; the $\mathrm{IC}_{50}$ of nab-paclitaxel in these cells was of $6.8 \mathrm{nM}$, about tenfold lower than those obtained in all other MM cells, either BRAF wt or mutated (Fig. 2a). The capability of this taxane to perturb cell cycle progression and to induce apoptosis was evaluated, and results, reported in Fig. 2, showed an accumulation of cells in G2/M phase (Fig. 2b) with a consequent induction of apoptosis (Fig. 2c), probably dependent from an impairment of mitochondria functionality as suggested by measurements of mitochondrial membrane depolarization (Fig. 2d). The evaluation of the involvement of Akt and Erk1/2 in determining the response to nab-paclitaxel was done by western blotting and showed that the drug did not impact on the survival and proliferation pathways (as reported in Fig. 2e), in agreement with data in literature demonstrating that nab-paclitaxel does not modify p-Akt and p-Erk1/2 levels $[13,14]$.

To explain the mechanism that might be responsible for the high effectiveness of nab-paclitaxel in MO- 1 cells, we explored the hypothesis recently published by Hertzman Johansson et al. who suggested that SILV/PMEL17/GP100 is transcriptionally regulated by MITF in melanocytes and melanoma, and consequently, a low expression level of MITF is responsible for the low level of SILV/PMEL17/ GP100 which in turn sensitizes cells to paclitaxel $[15,16]$. In MO-1 cells, characterized by a high sensitivity to nabpaclitaxel and a low expression level of MITF, also SILV/ PMEL17/GP100 was not consistently expressed; i.e., only 1.33-fold with respect to its isotype (in FCM analysis). Conversely, this factor was twofold and threefold higher in BRAF wild-type cells which showed high expression of MITF and low responsiveness to the drug. Thus, these results agreed with the hypothesis.

\section{Conclusions}

In conclusion, for the first time, we reported that in MM patients, the mutation G469A in BRAF gene, which in this disease has a frequency of $7 \%$, might be related to a high sensitivity to taxanes and a low effectiveness to BRAF inhibitors. Our study, extended to a large cell panel and validated in vivo, may lead to the optimization of therapy in MM.

Acknowledgments We thank Dr. I. Maida and Dr. S. Strippoli for collaboration in protein analysis and in patient's follow-up information, respectively.

Conflict of interest The authors have no conflict of interest.

\section{References}

1. Tentori L, Lacal PM, Graziani G (2013) Challenging resistance mechanisms to therapies for metastatic melanoma. Trends Pharmacol Sci 34:656-666. doi:10.1016/j.tips.2013.10.003

2. Wan PT, Garnett MJ, Roe SM, Lee S, Niculescu-Duvaz D, Good VM, Jones CM, Marshall CJ, Springer CJ, Barford D, Marais R, Cancer Genome Project (2004) Mechanism of activation of the RAF-ERK signaling pathway by oncogenic mutations of B-RAF. Cell 116:855-867

3. Kinno T, Tsuta K, Shiraishi K, Mizukami T, Suzuki M, Yoshida A, Suzuki K, Asamura H, Furuta K, Kohno T, Kushima R (2014) Clinicopathological features of nonsmall cell lung carcinomas with BRAF mutations. Ann Oncol 25:138-142. doi:10.1093/ annonc/mdt495

4. Paik PK, Arcila ME, Fara M, Sima CS, Miller VA, Kris MG, Ladanyi M, Riely GJ (2011) Clinical characteristics of patients with lung adenocarcinomas harboring BRAF mutations. J Clin Oncol 29:2046-2051. doi:10.1200/JCO.2010.33.1280

5. Flaherty KT, McArthur G (2010) BRAF, a target in melanoma: implications for solid tumor drug development. Cancer 116:4902-4913. doi:10.1002/cncr.25261

6. Lee MH, Lee SE, Kim DW, Ryu MJ, Kim SJ, Kim SJ, Kim YK, Park JH, Kweon GR, Kim JM, Lee JU, De Falco V, Jo YS, Shong M (2011) Mitochondrial localization and regulation of BRAFV600E in thyroid cancer: a clinically used RAF inhibitor is unable to block the mitochondrial activities of BRAFV600E. J Clin Endocrinol Metab 96:E19-E30. doi:10.1210/jc.2010-1071

7. Zanna P, Maida I, Turpin Sevilla MC, Susca FC, Filotico R, Arciuli M, Cassano N, Vena GA, Cicero R, Guida G (2011) Molecular characterization of novel melanoma cell lines. J Biol Regul Homeost Agents 25:239-247

8. Zanna P, Maida I, Grieco C, Guida S, Turpin Sevilla MC, De Summa S, Tommasi S, Vena GA, Filotico R, Guida G (2013) Three novel human sporadic melanoma cell lines: signaling pathways controlled by MC1R, BRAF and $\beta$-catenins. J Biol Regul Homeost Agents 27:131-141

9. Porcelli L, Quatrale AE, Mantuano P, Leo MG, Silvestris N, Rolland JF, Carioggia E, Lioce M, Paradiso A, Azzariti A (2013) 
Optimize radiochemotherapy in pancreatic cancer: PARP inhibitors a new therapeutic opportunity. Mol Oncol 7:308-322. doi:10.1016/j.molonc.2012.10.002

10. Azzariti A, Colabufo NA, Berardi F, Porcelli L, Niso M, Simone GM, Perrone R, Paradiso A (2006) Cyclohexylpiperazine derivative PB28, a sigma2 agonist and sigma1 antagonist receptor, inhibits cell growth, modulates P-glycoprotein, and synergizes with anthracyclines in breast cancer. Mol Cancer Ther 5:1807-1816

11. Porcelli L, Guida G, Quatrale AE, Cocco T, Sidella L, Maida I, Iacobazzi RM, Ferretta A, Stolfa DA, Strippoli S, Guida S, Tommasi S, Guida M, Azzariti A (2015) Aurora kinase B inhibition reduces the proliferation of metastatic melanoma cells and enhances the response to chemotherapy. J Transl Med 13:26. [Epub ahead of print]

12. Velho TR (2012) Metastatic melanoma-a review of current and future drugs. Drugs Context 2012:212242. doi:10.7573/ dic.212242 (eCollection 2012)
13. Rajput S, Volk-Draper LD, Ran S (2013) TLR4 is a novel determinant of the response to paclitaxel in breast cancer. Mol Cancer Ther 12:1676-1687. doi:10.1158/1535-7163.MCT-12-1019

14. Li S, De Souza P (2011) Ras isoprenylation and pAkt inhibition by zoledronic acid and fluvastatin enhances paclitaxel activity in T24 Bladder cancer cells. Cancers (Basel) 3:662-674. doi:10.3390/cancers3010662

15. Du J, Miller AJ, Widlund HR, Horstmann MA, Ramaswamy S, Fisher DE (2003) MLANA/MART1 and SILV/PMEL17/GP100 are transcriptionally regulated by MITF in melanocytes and melanoma. Am J Pathol 163:333-343

16. Hertzman Johansson C, Azimi A, Frostvik Stolt M, Shojaee S, Wiberg H, Grafström E, Hansson J, Egyházi Brage S (2013) Association of MITF and other melanosome-related proteins with chemoresistance in melanoma tumors and cell lines. Melanoma Res 23:360-365 